\title{
Biomateriais usados na revascularização pulpar: revisão de literatura
}

\author{
Biomaterials used in pulp revascularization: literature review \\ Biomateriales utilizados em revascularización pulpar: revisión de la literatura
}

Recebido: 15/01/2021 | Revisado: 20/01/2021 |Aceito: 24/01/2021 | Publicado: 31/01/2021

Nathália Evelyn da Silva Machado
ORCID: https://orcid.org/0000-0002-4196-782X
Universidade Estadual Paulista, Brasil
E-mail: nathalia.machao@unesp.br
Vitória Marques Gomes
ORCID: https://orcid.org/0000-0002-9709-8598
Universidade Estadual Paulista, Brasil
E-mail: vitória.m.gomes @unesp.br
Ana Maria Veiga Vasques
ORCID: https://orcid.org/0000-0002-1211-2363
Universidade Estadual Paulista, Brasil
E-mail: anavvasques @hotmail.com
Ana Paula Fernandes Ribeiro
ORCID: https://orcid.org/0000-0001-5313-5003
Universidade Estadual Paulista, Brasil
E-mail: ana_paulafernandesr@ hotmail.com
Anderson Maikon de Souza Santos
ORCID: https://orcid.org/0000-0001-9371-9417
Universidade Estadual Paulista, Brasil
E-mail: andersonmaikon @ hotmail.com
Bruno Coelho Mendes
ORCID: https://orcid.org/0000-0001-7896-8909
Universidade Estadual Paulista, Brasil
E-mail: brunocoelho.mnds@ @mail.com
Luciano Tavares Ângelo Cintra
ORCID: https://orcid.org/0000-0003-2348-7846
Universidade São Paulo, Brasil
E-mail: luciano.cintra @unesp.br
Ericke Mucke da Silva
ORCID: https://orcid.org/0000-0002-4540-7328
Universidade São Paulo, Brasil
E-mail: ericke_mucke@ hotmail.com

\section{Resumo}

Fundamento: durante muitas décadas a apicificação foi o tratamento preconizado para dentes permanentes imaturos com polpas não-vitais. Atualmente, tem-se buscado a revitalização destes dentes através da revascularização pulpar. Esta técnica permite que os dentes permanentes imaturos com polpas não-vitais completem o desenvolvimento da raiz, e utiliza biomateriais para esta finalidade. O MTA é um cimento de silicato de cálcio, e atualmente, é o biomaterial mais indicado para o selamento cervical na técnica de revascularização pulpar, porém ele apresenta desvantagens. Novos biomateriais estão sendo desenvolvidos para tentar melhorar algumas propriedades do MTA. Objetivo: o objetivo deste estudo foi realizar uma revisão da literatura comparando as propriedades físicas, químicas e biológicas do MTA com os novos biomateriais que estão surgindo no mercado, para emprego na revascularização pulpar. Conclusão: na literatura foi encontrado o uso de biomateriais como o Endosequence e o Biodentine na revascularização pulpar de dentes permanentes imaturos com polpa não-vital, porém até o momento não existem trabalhos clínicos randomizados que comprovem as vantagens destes materiais em relação ao MTA.

Palavras-chave: Materiais biocompatíveis; Agentes indutores da angiogênese; Endodontia; Endodontia regenerativa; Hidróxido de cálcio.

\footnotetext{
Abstract

Background: for many decades apexification was the recommended treatment for immature permanent teeth with nonvital pulps. Currently, the revitalization of these teeth has been sought through pulp revascularization. This technique allows immature permanent teeth with non-vital pulps to complete root development, and uses biomaterials for this purpose. MTA is a calcium silicate cement, and is currently the most suitable biomaterial for cervical sealing in the pulp revascularization technique, but it has disadvantages. New biomaterials are being developed to try to improve some properties of the MTA. Objective: the aim of this study was to conduct a literature review comparing the physical, chemical and biological properties of MTA with the new biomaterials that are emerging on the market, for
} 
use in pulp revascularization. Conclusion: in the literature, the use of biomaterials such as Endosequence and Biodentine was found in pulp revascularization of immature permanent teeth with non-vital pulp, however, to date, there are no randomized clinical studies that prove the advantages of these materials in relation to MTA.

Keywords: Biocompatible materials; Angiogenesis inducing agents; Endodontics; Regenerative endodontics; Calcium hydroxide.

\section{Resumen}

Antecedentes: durante muchas décadas, la apexificación fue el tratamiento recomendado para los dientes permanentes inmaduros con pulpas no vitales. Actualmente, se ha buscado la revitalización de estos dientes mediante la revascularización pulpar. Esta técnica permite que los dientes permanentes inmaduros con pulpas no vitales completen el desarrollo radicular y utiliza biomateriales para este propósito. El MTA es un cemento de silicato de calcio, y actualmente es el biomaterial más adecuado para el sellado cervical en la técnica de revascularización pulpar, pero tiene desventajas. Se están desarrollando nuevos biomateriales para intentar mejorar algunas propiedades del MTA. Objetivo: el objetivo de este estudio fue realizar una revisión de la literatura comparando las propiedades físicas, químicas y biológicas del MTA con los nuevos biomateriales que están surgiendo en el mercado, para su uso en la revascularización pulpar. Conclusión: en la literatura se encontró el uso de biomateriales como Endosequence y Biodentine en la revascularización pulpar de dientes permanentes inmaduros con pulpa no vital, sin embargo, a la fecha no existen estudios clínicos aleatorizados que demuestren las ventajas de estos materiales en relación al MTA.

Palabras clave: Materiales biocompatibles; Agentes inductores de angiogénesis; Endodoncia; Endodoncia regenerativa; Hidróxido de cálcio.

\section{Introdução}

Os dentes permanentes imaturos podem tornar-se não-vitais, geralmente como resultado de traumatismos dentários, cárie ou anormalidades congênitas (He et al., 2017). Para o tratamento destes casos, durante muitas décadas, indicou-se a apicificação. Este é um procedimento onde o canal radicular é instrumentado e são realizadas várias trocas da medicação intracanal, até que seja confirmada por meio de radiografias a formação de um tecido calcificado, permitindo o fechamento apical de forma artificial, por meio da formação de uma barreira mineralizada estimulada por um biomaterial (Damle et al., 2012; Diogenes et al., 2013; Bezgin et al., 2015; Songtrakul et al., 2020). Nessa técnica são necessários vários retornos para o controle e a troca da medicação intracanal; além das desvantagens de causar injúrias às paredes dentinárias das raízes que não estão completamente formadas (Bezgin et al., 2015; Songtrakul et al., 2020). Diversos trabalhos demonstraram que este tratamento que não promove o desenvolvimento radicular, e sim, o fechamento apical (Damle et al., 2012; Diogenes et al., 2013; Albuquerque et al., 2014; Hargreaves et al., 2013; Songtrakul et al., 2020).

Como uma alternativa para suprir as desvantagens da apicificação surgiu a revascularização pulpar; esta é um procedimento simples e de baixo custo, tendo como principal objetivo induzir o crescimento radicular, aumentando a espessura da parede radicular (Songtrakul et al., 2020; Bezgin et al., 2015; Albuquerque et al., 2014; Hargreaves et al., 2013; Palit et al., 2014; Murray et al., 2007).

A revascularização pulpar permite que os dentes permanentes imaturos com polpas não-vitais completem o desenvolvimento radicular (He et al., 2017; Songtrakul et al., 2020). O tratamento é direcionado à realização de protocolos de desinfecção dos canais radiculares na primeira sessão, sendo indicada irrigação com hipoclorito de sódio seguido do uso de uma medicação intracanal, que pode ser uma pasta de hidróxido de cálcio ou uma combinação de antibióticos como ciprofloxacina, metronidazol e minociclina, que também é conhecida como pasta triantibiótica. Na segunda sessão é realizada a indução de um coágulo sanguíneo e feito um selamento cervical com um biomaterial, pois seu mecanismo de ação tem o potencial de interagir com células-tronco devido à sua capacidade osteocondutora (Torabinejad er al., 2010; Kontakiotis et al., 2015; Songtrakul et al., 2020), estimulando assim o desenvolvimento radicular. Um dos exemplos de biomateriais são os cimentos à de silicato de cálcio (Araújo et al., 2017; Ürkmez and Erdem. 2020). O procedimento de revascularização possui maiores taxas de sucesso quando comparado à apicificação, pois além de completar o desenvolvimento radicular, favorece o espessamento das paredes dentinárias (Chaniotis et al. 2017).

Para haver a estimulação nos tecidos do complexo dentino-pulpar e manter a viabilidade das células na região apical, 
os biomateriais utilizados necessitam apresentar algumas características, sendo elas, ser biocompatível com os tecidos periapicais por estarem em íntimo contato com os mesmos; ser radiopaco, pela necessidade de visualização radiográfica do material, apresentar um bom selamento para que não ocorra recontaminação dos canais radiculares, e finalmente, apresentar uma boa estabilidade físico-química para manter o ambiente apropriado para preservação das células, e consequentemente a regeneração tecidual (Sinsek et al., 2015; Ürkmez and Erdem. 2020). O MTA é portanto o material indicado atualmente para a revascularização pulpar, entretanto, novos biomateriais estão sendo desenvolvidos para tentar melhorar algumas propriedades do MTA, superando suas desvantagens. MTA Plus, NeoMTA Plus, Biodentine e Endosequence são alguns desses materiais que vem sendo utilizados para selamento cervical na revascularização pulpar, e parecem ser biomateriais promissores.

Este estudo teve como objetivo, por meio de uma revisão de literatura, verificar e comparar o uso do MTA e outros biomateriais para selamento cervical no tratamento de revascularização pulpar.

\section{Metodologia}

Para este estudo foram revisados artigos encontrados nas bases de dados PubMed/MEDLINE, SCOPUS e Cochrane Library. As palavras-chave utilizadas na busca foram "Mineral Trioxide Aggregate", "Revascularization", "Root Canal Filling Materials" e "Endodontics". No momento da busca as palavras foram cruzadas a cada duas, três e após todas juntas para uma pesquisa mais fidedigna sobre o assunto; o unitermo de escolha foi "AND", porque o objetivo era agrupar os assuntos, e não distinguir entre um e outro, além de não ter sido utilizado nenhum tipo de filtro. É importante relatar que só foram incluídos artigos publicados em inglês e até o período de dezembro de 2020.

\section{Revisão de Literatura e Discussão}

\section{MTA como biomaterial utilizado na endodontia regenerativa}

O Mineral Trióxido Agregado (MTA) é um cimento de silicato de cálcio (Tomás-Catalá et al., 2017) baseado no cimento Portland, que foi desenvolvido há mais de 20 anos, na década de 90 (Kahler et al., 2017). É um biomaterial indicado em diversas aplicações clínicas odontológicas, principalmente a terapia endodôntica (Soboni et al., 2017; Aly et al., 2019; Quintana et al., 2019).

Ele é constituído por um pó de partículas finas hidrofílicas que necessitam de presença de umidade para solidificar (Roberts et al., 2008). Com isso, seu uso é indicado em perfurações nos canais radiculares, perfurações na furca, reabsorções radiculares, na cirurgia parendodôntica como material retro-obturador, pulpotomia, apicigênese, apicificação, proteção pulpar direta, base de cavidade para selamento coronário e cimento obturador. Além disso, atualmente também vem sendo utilizado na revascularização pulpar realizada em dentes imaturos com ápice aberto (Bhola et al., 2017; Quintana et al 2019; Aly et al., 2019). Neste procedimento regenerativo há evidências de formação de barreira apical mineralizada e redução no tempo de tratamento, obtendo resultados de cura favoráveis (Kahler et al., 2017).

Possui em sua composição 75\% de cimento Portland, 20\% de óxido de bismuto e 5\% de gesso (Bhola et al., 2017; Kaur et al., 2017; Patidar et al., 2017; Camilleri et al., 2015) e após sua manipulação é formado silicato dicálcico e tricálcico, aluminato tricálcico, óxido tricálcico e óxido de silicato (Tompas-Catalá et al., 2017; Naghavi et al., 2014; Paritok et al., 2010). É um material radiopaco, biocompatível e não sofre contração. Além de não ser sensível à umidade (com exceção da saliva) e à contaminação sanguínea. Outras características importantes do MTA são a capacidade de promover um selamento eficaz, reparação biológica e regeneração do ligamento periodontal (Saeki et al., 2014; Kim et al., 2006). Ainda, é considerado um material bioativo porque induz a formação de tecido duro na região apical (Bhola et al., 2017).

Seu potencial para novas aplicações clínicas tornou-se evidente devido às suas propriedades químicas e habilidade de selamento (Prati et al., 2015; Bakland et al., 2012). Esse biomaterial quando em contato com os flúidos teciduais ocorre a 
hidratação e formação de hidróxido de cálcio, com isso promove a liberação de íons de cálcio e hidroxila que são essenciais para a diferenciação e proliferação celular, podendo regenerar e mineralizar os tecidos duros da região. Além disso, melhora a capacidade de selamento da área, porque deposita cristais de cálcio nos espaços vazios entre a dentina e o material de preenchimento radicular, possui uma boa solubilidade e um alto $\mathrm{pH}$, criando assim um ambiente antimicrobiano (Tomás-Catalá et al., 2017; Siboni et al., 2017; Parirok et al., 2010).

O MTA tem demonstrado melhores características biocompatíveis que outros biomateriais. Com relação à indução de tecidos mineralizados em dentes permanentes imaturos com polpa não-vital, o seu maior índice de sucesso pode estar relacionado com alguns fatores. Primeiramente, que o MTA é um material indutor de tecido cementário (cemento), com isso a deposição de tecido mineralizado se torna favorável, e possui capacidade de selamento hermético, impedindo a entrada de microrganismos (Bakland et al., 2012; Aly et al., 2019).

Quando colocado em contato direto com os tecidos, o MTA libera íons cálcio, o que ativa as células da região mantendo o pH elevado. Dessa maneira, cria um ambiente antimicrobiano (Bakland et al., 2012). Ademais, seu pH é mutável, ou seja, é variável dependendo da área onde está inserido, no momento da sua aplicação apresenta pH próximo a 10, e 3 horas após sua aplicação aumenta para 12 (Kim et al., 2006; Quintana et al., 2019).

Alguns estudos demonstraram que os cimentos à base de MTA, que tem como principal componente o silicato tricálcio, não são genotóxicos e/ou citotóxicos ás células da polpa dentária (De-Deus et al., 2009; Bortoluzzi et al., 2015; Agrafioti et al., 2016; Peters et al. 2016).

Apesar de apresentar excelentes propriedades químicas, físicas e biológicas, muitas vezes o MTA deixa de ser utilizado por clínicos devido à sua dificuldade de manipulação e seu longo tempo de presa (Lee et al., 2014). Além disso, possui uma grande desvantagem, que é o manchamento da coroa dos dentes nos quais foi utilizado (Kahler et al., 2017; Kaur et al., 2017; Patidar et al., 2017; Lee et al., 2014;Carmen et al., 2017; Aly et al., 2019).

Na revascularização pulpar, ocorre formação de pontes de cemento devido à sua capacidade de osteocondução (Tanomaru-Filho et al., 2017; Aly et al., 2019).

\section{MTA Plus}

Como já foi citado anteriormente, apesar do MTA apresentar excelentes qualidades, ele demonstra algumas desvantagens, dentre elas sua dificuldade de manipulação, impossibilidade de ser exposto a fluidos orais e possibilidade de manchamento da estrutura dentária (Soboni et al., 2017; Quintana et al., 2019). Com isso foram lançadas algumas modificações do MTA para tentar superar tais desvantagens. A primeira delas foi o MTA Plus, também chamado de MTA Branco (Camilleri et al., 2015).

Os componentes do pó do MTA Plus são silicato tricálcio e óxido de bismuto, e o líquido é composto por um gel de água destilada e um outro componente que não foi liberado pelo fabricante (Tomás-Catalá et al., 2017). O óxido de bismuto é muito importante na composição deste biomaterial uma vez que ele atua no processo de hidratação do silicato de cálcio, porém, produz manchamento da coroa dentária (Tomás-Catalá et al., 2017; Siboni et al., 2017). Em estudo realizado por Camilleri et al., em 2015, verificou-se que quando em contato com um agente irrigante, especialmente o hipoclorito de sódio, que é o mais utilizado em tratamentos endodônticos, este efeito indesejável é apresentado (Camilleri et al., 2015). Nos casos de revascularização é comum o uso de hipoclorito de sódio na concentração de 5,25\%, o que provavelmente traria as alterações de manchamento já citadas.

O mecanismo de ação do MTA Plus na endodontia regenerativa, dá-se de maneira semelhante à do seu precursor. Quando do contato íntimo do material com a dentina da câmara pulpar, há a estimulação e produção/ liberação de moléculas de sinalização, que são necessárias para a formação de novos tecidos no espaço da polpa, estimulando assim o desenvolvimento 
radicular (Songtrakul et al., 2020).

\section{NeoMTA Plus}

Devido às desvantagens do MTA Plus (Avalon Biomed Inc., Bradenton, FL, USA), foi proposta uma mudança na composição da sua porção em pó, já que um de seus componentes, o óxido de bismuto, seria o responsável pelo manchamento dos dentes. Foi então lançado o NeoMTA Plus (Avalon Biomed, Houston, TX, USA), uma modificação do MTA Plus na qual o agente radiopacificador óxido de bismuto foi substituído pelo óxido de tântalo (Ta2O5). A composição do líquido foi mantida igual, e a vantagem da modificação da composição do pó foi, principalmente, a obtenção de um melhor tempo de trabalho para o uso em preenchimento de canais radiculares e a diminuição de manchamento nos dentes em que foi empregado (Ürkmez and Erdem. 2020; Siboni et al., 2017; Tomás-Catalá et al., 2017), sendo considerado uma alternativa estética ao MTA convencional e MTA Plus (Patil et al., 2019).

O potencial descolorante dos materias de silicato de cálcio que não contém óxido de bismuto foi considerado menor (Camilleri et al., 2014; Keskin et al., 2015), porém a longo prazo relataram descoloração clinicamente perceptível induzida por esses biomateriais (Mozynska eu al., 2017). A hipótese de que os agentes clareadores alteram a resistência à compressão do MTA Plus e do NeoMTA Plus foi testada, e os resultados mostraram que ambos os biomateriais quando em contato com os agentes clareadores tiverem sua resistência à compressão diminuídas, sendo assim, quando houver a necessidade de se realizar clareamento por conta do manchamento nos dentes, os biomateriais devem ser complemente removidos da porção coronária (Keskin et al., 2019).

Segundo trabalho de Tanomaru-Filho, a substituição do óxido de bismuto por Ta2O5 não alterou a biocompatibilidade e a bioatividade do novo cimento. O tântalo é um biomaterial com diversas aplicações, especialmente na regeneração de tecidos, e possui excelente radiopacidade com baixa citotoxicidade (Tanomaru-Filho et al., 2017) .

Em estudo realizado por Siboni et al em 2017, foi comprovado que o NeoMTA Plus apresenta uma maior e prolongada liberação de íons de cálcio e hidroxila quando comparado com o MTA Plus permitindo maior capacidade do material em formar uma camada de fosfato de cálcio (Siboni et al., 2017).

Assim como a maioria dos BECs, o NeoMTA Plus tem o potencial de interagir com células-tronco devido à sua capacidade osteocondutora (Torabinejad et al., 2010; Kontakioyis et al., 2015). Além disso, algumas investigações laboratoriais demonstraram a bioatividade dos BECs e sua condutividade para formação de tecido duro (Parirokh et la., 2018), que são considerados pré-requisitos em casos de revascularização.

Apesar das vantagens dos procedimentos de revascularização em comparação com a colocação de tampões apicais de MTA ou BECs já terem sido descritas por estudos que demonstraram um fechamento apical mais reforçado, maior comprimento e espessamento da raiz (Narang et al., 2015), ainda não há estudos com resultados a longo prazo em humanos, sendo necessário maior número de investigações para que esta possa ser uma prática clínica consolidada.

\section{Biodentine}

Biodentine é um cimento reparador de silicato tricálcio (Ca3SiO5) baseado na composição do cimento Portland e desenvolvido na tentativa do fabricante de melhorar suas propriedades fisico-químicas e biológicas. O produto consiste de um pó (silicato tricálcio, carbonato de cálcio e óxido de zircônia como radiopacificador) e um líquido (contendo cloreto de cálcio e um polímero solúvel em água) que são misturados para formar o cimento (Nai et al., 2016; Quintana et al., 2019).

Materiais utilizados em procedimentos como terapias vitais da polpa, terapias endodônticas regenerativas ou reparações de perfuração, devem possuir, dentre outras propriedades, biocompatibilidade. Biodentine demonstrou ser biocompatível quando testado em vários tipos de células, apresentando melhores propriedades de manipulação e um tempo de 
endurecimento mais curto quando comparado com o MTA (Agrafioti et al., 2016; Pradelle-Plasse \& Tran Xuan-Vin, 2009). Apresenta uma resposta inflamatória nos primeiros 7 dias e histologicamente há presença de células inflamatórias que reduzem significativamente após 14 dias, caracterizando sua biocompatibilidade. Um material que permite uma redução na resposta inflamatória ao longo do tempo é considerado biocompatível, a exemplo do que ocorre com o hidróxido de cálcio. Ele promove uma resposta inflamatória inicial quando em contato com os tecidos, e após poucos dias ela torna-se insignificante, ocorrendo o reparo (Mori et al., 2014; Silva et al., 2016).

Biodentine é citocompatível de acordo com ensaios realizados em cultura de células 3D (Odabas et al., 2013), e apresenta maior resiliência à força de cisalhamento do que outros cimentos (Laurent et al., 2008). Além disso, o Biodentine tem a habilidade de induzir a diferenciação odontoblástica e a mineralização (Laurent et al., 2012), e quando aplicado diretamente sobre o tecido pulpar induz uma dentinada reparadora inicial devido a uma modulação na secreção de TGF- $\beta 1$ das células pulpares (Raiase Kharan et al., 2018).

Outras propriedades importantes do Biodentine são alta resistência à compressão, possui altos valores de microdureza, boa resistência flexural e boa capacidade de vedação (Namour \& Theys, 2014). Devido às suas boas propriedades físicoquímicas e biológicas, é recomendado como substituto da dentina podendo ser usado para a mesma finalidade que o MTA (Agrafioti et al., 2016; Ürkmez and Erdem. 2020). É um material potencial para proteção do coágulo sanguíneo no procedimento de revascularização pulpar devido à sua consistência, facilidade de manipulação e composição (Bortoluzzi et al., 2015). Penetra através dos túbulos dentinários abertos e cristaliza quando se entrelaça com a dentina, proporcionando suas propriedades mecânicas (Kaur et al., 2017).

O sucesso do tratamento de revascularização pulpar depende de três fatores: desinfecção do canal radicular, presença de um coágulo sanguíneo e selamento cervical hermético (Sawyer et al., 2012). O MTA demonstrou resultados melhores que Biodentine com relação à capacidade de evitar movimentos dos fluidos quando utilizado em ambientes com pH neutro. Porém, quando em contato com um $\mathrm{pH}$ ácido, ambos os biomateriais apresentaram resultados semelhantes e satisfatórios de capacidade de selamento (Agrafioti et al., 2016).

Em um estudo realizado por Aly et al, em 2019 verificou clinicamente e radiograficamente a evolução de dentes permanentes imaturos tratados com procedimento de revascularização, sendo um estudo randomizado. Nele foi possível realizar o tratamento e acompanhar dentes permanentes imaturos onde realizou-se o procedimento regenerativo com o biodentine e o MTA, e por fim, comparar os materiais utilizados. Os autores concluíram que o biodentine apresentou as mesmas propriedades do MTA, e superou as desvantagens clínicas do mesmo; apresentando uma melhor consistência, permitindo que a restauração fosse realizada na mesma sessão e uma menos descoloração da coroa dentária (Aly et al., 2019).

Biodentine possui as mesmas propriedades mecânicas que a dentina, sofre expansão e preenche todo o espaço devido à sua plasticidade (De Souza et al., 2013; Aly et al., 2019). Outra vantagem é que ao contrário do MTA, não causa pigmentação da área cervical (Sawyer et al., 2012). De Souza et al., em seu estudo sobre porosidade compararam Biodentine com ProRoot MTA e observaram que não houve diferença significativa entre os materiais (De Souza et al., 2013).

Em um relato de 3 casos clínicos nos quais foi realizado o tratamento de revascularização pulpar seguindo o mesmo protocolo, variando apenas o tipo de biomaterial utilizado, o autor verificou insucesso em todos os casos, independente do biomaterial utilizado. Estas falhas foram associadas à provável permanência de microrganismos intracanais após a conclusão dos protocolos de desinfecção em uma única sessão. Isto demonstra que se a descontaminação dos canais radiculares não for efetiva, não haverá diferença entre o uso de MTA ou Biodentine para selamento cervical na revascularização pulpar (Chaniotis et al., 2017).

MTA tem apresentado resultados favoráveis com relação à citotoxicidade e os achados na literatura revelam que Biodentine tem demonstrado resultados semelhantes. (Bortoluzzi et al., 2015; Khedmat et al., 2014; Jung et al., 2015; Aly et 
al., 2019). Na viabilidade celular, ambos os biomateriais obtiveram atividades enzimáticas mitocondriais equivalentes, e em relação ao potencial osteogênico. Biodentine demonstrou induzir significativamente mais formação de nódulos mineralizados do que MTA (Zanini et al., 2012).

Joshi et al. (2020) analisando casos clínicos na literatura, avaliaram o potencial efeito do MTA e Biodentine quando associados a uma fibrina rica em plaquetas, utilizada como barreira apical junto com os biomateriais, nos tratamentos de revascularização de dentes permanentes imaturos. Como resultado, o preenchimento ósseo se deu de maneira mais rápida, uma vez que o MTA e o Biodentine auxiliram na chamada reparação tecidual guiada, e na regeração óssea.

Entretanto, Nai et al. observaram DNA danificado no teste do micronúcleo, sugerindo um potencial mutagênico, podendo ser clastogênico ou aneugênico. Por outro lado não foram demonstrados efeitos genotóxicos no teste do cometa. Não há na literatura estudos in vivo avaliando a exposição a diferentes doses de Ca3SiO5 (Nai et al., 2016).

Existe na literatura uma quantidade considerável de estudos sobre Biodentine e suas propriedades, porém há a necessidade de realização de mais estudos que avaliem sua utilização na revascularização pulpar, principalmente estudos clínicos randomizados, que são uma poderosa ferramenta para a avaliação de intervenções para a saúde.

\section{Endosequence Root Repair}

Endosoquence Root Repair Putty (Brasseler USA, Savannah, GA) e Endosequence Root Repair Paste (Brasseler EUA) foram desenvolvidos como materiais biocerâmicos pré-misturados prontos para uso, indicados para selamento de perfurações, cirurgia parendodôntica e selamento apical e coronário. De acordo com o fabricante, ambos os materiais são de composição química bastante semelhante (silicatos de cálcio, óxido de zircônia, óxido de tântalo e fosfato de cálcio monobásico). Eles apresentam excelentes propriedades físicas e biológicas e são fáceis de trabalhar. São hidrofílicos, insolúveis, radiopacos, sem alumínio e possuem pH elevado (Lee et al., 2014).

Endosequence Root Repair Putty tem sido utilizado com sucesso no selamento coronário em procedimentos de revascularização pulpar em dentes permanentes imaturos com polpas não-vitais, dentes com invaginação, reabsorção cervical invasiva e após avulsão dentária (Torabinejad et al., 2010). Além disso, este biomaterial obteve maior formação de osso, cemento e ligamento periodontal cobrindo as superfícies do ápice radicular quando comparado ao ProRoot MTA (Torabinejad et al., 2018; Chen et al., 2015).

Os cimentos biocerâmicos e o MTA demonstraram uma semelhança referente à biocompatibilidade, atividade antifúngica e antibacteriana.(De-Deus et al., 2009; Lee et al., 2014).

Muitos pesquisadores demonstraram que a qualidade e a quantidade de adesão celular aos materiais utilizados na terapia regenerativa podem ser vistas como critério para a avaliação da biocompatibilidade dos mesmos (Zhu et al., 2012). Assim como o MTA, os cimentos Endosequence Root Repair Putty e Endosequence Root Repair Paste não impediram a adesão celular, apesar de possuírem cristais na sua superfície. As células se encontraram bem espalhadas na superfície do material e proliferaram, demonstrando uma resposta celular favorável aos materiais (Lee et al., 2014).

Algumas investigações não relataram diferença significante na utilização de MTA ou Endosequence como material de preencimento da raiz em cirurgias parendodônticas (Torabinejad et al., 2018; Zhou et al., 2017).

Ma et al. avaliaram a citotoxicidade dos cimentos biocerâmicos Endosequence Root Repair Putty e Endosequence Root Repair comparados ao MTA durante intervenções de revascularização em dentes permanents imaturos com polpa nãovital, demonstrando que os mesmos exibiram viabilidade celular semelhantes ao MTA em todas as condições experimentais, exceto em amostras com menos de 7 dias de Endosequence Root Repair Paste, que apresentou viabilidade celular ligeiramente mais baixa do que o MTA (Ma et al., 2011).

Casos de revascularização pulpar em dentes com rizogênese incompleta nos quais foram empregados EndoSequence 
Root Repair Putty ou MTA, aleatoriamente, obtiveram resultados positivos, indicando que tanto o MTA, quanto o cimento biocerâmico podem ser utilizados com sucesso (Buknari et al., 2016). Embora a correlação clara entre a biocompatibilidade in vitro e in vivo já tenha sido descrita para o MTA, são necessárias novas pesquisas para EndoSequence Putty e EndoSequence Paste, aplicando testes como os de genotoxicidade e sensibilização para que se estabeleça uma visão mais ampla sobre a aplicação clínica destes materiais (Lee et al., 2014).

Embora os estudos realizados com os cimentos biocerâmicos Endosequence Root Repair tenham demonstrado resultados promissores em aplicações clínicas regenerativas quando comparados com o MTA, o número de investigações que avaliam suas propriedades físicas, químicas e biológicas ainda é pequeno, e a sua eficácia a longo prazo ainda é desconhecida. Portanto, mais pesquisas com altos níveis de evidência e metodologias rigorosas são necessárias (Torabinejad et al., 2018).

\section{Conclusão}

$\mathrm{Na}$ literatura há a indicação do uso de biomateriais, como o Endosequence e Biodentine na revascularização pulpar de dentes permanentes imaturos com polpa não-vital, porém até o momento não existem trabalhos clínicos randomizados que comprovem as vantagens destes materiais em relação ao MTA. Há a necessidade de realização destes estudos, considerando serem os mais apropriados para se avaliar o uso dos biomateriais estudados.

\section{Referências}

Agrafioti, A., Tzimpoulas, N., Chatzitheodoridis, E., \& Kontakiotis, E. G. (2016). Comparative evaluation of sealing ability and microstructure of MTA and Biodentine after exposure to different environments. Clin Oral Investig, 20(7), 1535-1540.

Albuquerque, M. T., Valera, M. C., Nakashima, M., Nör, J. E., \& Bottino, M. C. (2014). Tissue-engineering-based strategies for regenerative endodontics. Journal of dental research, 93(12), 1222-1231.

Aly, M. M., Taha, S., El Sayed, M. A., Youssef, R., \& Omar, H. M. (2019). Clinical and radiographic evaluation of Biodentine and Mineral Trioxide Aggregate in revascularization of non-vital immature permanent anterior teeth (randomized clinical study). International journal of paediatric dentistry, 29(4), $464-473$.

Araújo, P., Silva, L. B., Neto, A., Almeida de Arruda, J. A., Álvares, P. R., Sobral, A., Júnior, S. A., Leão, J. C., Braz da Silva, R., \& Sampaio, G. C. (2017). Pulp Revascularization: A Literature Review. The open dentistry journal, 10, 48-56.

Bakland, L. K., \& Andreasen, J. O. (2012). Will mineral trioxide aggregate replace calcium hydroxide in treating pulpal and periodontal healing complications subsequent to dental trauma? A review. Dental traumatology: official publication of International Association for Dental Traumatology, 28(1), 25-32.

Bezgin, T., \& Sönmez, H. (2015). Review of current concepts of revascularization/revitalization. Dental traumatology: official publication of International Association for Dental Traumatology, 31(4), 267-273.

Bhola, M., Goyal, V., Tyagi, P., \& Kumar, T. (2017). Mineral trioxide aggregate-induced apical closure in nonvital immature permanent maxillary incisor. Journal of the Indian Society of Pedodontics and Preventive Dentistry, 35(4), 378-380.

Bortoluzzi, E. A., Niu, L. N., Palani, C. D., El-Awady, A. R., Hammond, B. D., Pei, D. D., Tian, F. C., Cutler, C. W., Pashley, D. H., \& Tay, F. R. (2015). Cytotoxicity and osteogenic potential of silicate calcium cements as potential protective materials for pulpal revascularization. Dental materials: official publication of the Academy of Dental Materials, 31(12), 1510-1522.

Bukhari, S., Kohli, M. R., Setzer, F., \& Karabucak, B. (2016). Outcome of Revascularization Procedure: A Retrospective Case Series. Journal of endodontics, 42(12), 1752-1759.

Camilleri J. (2015). Staining Potential of Neo MTA Plus, MTA Plus, and Biodentine Used for Pulpotomy Procedures. Journal of endodontics, 41(7), 11391145 .

Carmen, L., Asunción, M., Beatriz, S., \& Rosa, Y. V. (2017). Revascularization in Immature Permanent Teeth with Necrotic Pulp and Apical Pathology: Case Series. Case reports in dentistry, 2017, 3540159.

Camilleri J. (2014). Color stability of white mineral trioxide aggregate in contact with hypochlorite solution. Journal of endodontics, 40(3), 436-440.

Chaniotis A. (2017). Treatment Options for Failing Regenerative Endodontic Procedures: Report of 3 Cases. Journal of endodontics, $43(9)$, 1472-1478.

Chen, I., Karabucak, B., Wang, C., Wang, H. G., Koyama, E., Kohli, M. R., Nah, H. D., \& Kim, S. (2015). Healing after root-end microsurgery by using mineral trioxide aggregate and a new calcium silicate-based bioceramic material as root-end filling materials in dogs. Journal of endodontics, 41(3), 389-399.

Damle, S. G., Bhattal, H., \& Loomba, A. (2012). Apexification of anterior teeth: a comparative evaluation of mineral trioxide aggregate and calcium hydroxide paste. The Journal of clinical pediatric dentistry, 36(3), 263-268. 
De Souza, E. T., Nunes Tameirão, M. D., Roter, J. M., De Assis, J. T., De Almeida Neves, A., \& De-Deus, G. A. (2013). Tridimensional quantitative porosity characterization of three set calcium silicate-based repair cements for endodontic use. Microscopy research and technique, 76(10), $1093-1098$.

De-Deus, G., Canabarro, A., Alves, G., Linhares, A., Senne, M. I., \& Granjeiro, J. M. (2009). Optimal cytocompatibility of a bioceramic nanoparticulate cement in primary human mesenchymal cells. Journal of endodontics, 35(10), 1387-1390.

Diogenes, A., Henry, M. A., Teixeira, F. B., \& Hargreaves, K. M. (2013). An update on clinical regenerative endodontics. Endodontic Topics, 28 (1), $2-28$.

Hargreaves, K. M., Diogenes, A., \& Teixeira, F. B. (2013). Treatment options: biological basis of regenerative endodontic procedures. Journal of endodontics, 39(3 Suppl), S30-S43.

He, L., Zhong, J., Gong, Q., Kim, S. G., Zeichner, S. J., Xiang, L., Ye, L., Zhou, X., Zheng, J., Liu, Y., Guan, C., Cheng, B., Ling, J., \& Mao, J. J. (2017). Treatment of Necrotic Teeth by Apical Revascularization: Meta-analysis. Scientific reports, 7(1), 13941.

Joshi, S. R., Palekar, A. U., Pendyala, G. S., Mopagar, V., Padmawar, N., \& Shah, P. (2020). Clinical Success of Platelet-rich Fibrin and Mineral Trioxide Aggregate (MTA) or MTA-like Agents in Healing of Periapical Lesion in Nonsurgically Treated Pulpless Immature Permanent Teeth: A Systematic Review. Journal of International Society of Preventive \& Community Dentistry, 10(4), 379-383.

Jung, J. Y., Woo, S. M., Lee, B. N., Koh, J. T., Nör, J. E., \& Hwang, Y. C. (2015). Effect of Biodentine and Bioaggregate on odontoblastic differentiation via mitogen-activated protein kinase pathway in human dental pulp cells. International endodontic journal, 48(2), 177-184.

Kahler, B., Rossi-Fedele, G., Chugal, N., \& Lin, L. M. (2017). An Evidence-based Review of the Efficacy of Treatment Approaches for Immature Permanent Teeth with Pulp Necrosis. Journal of endodontics, 43(7), 1052-1057.

Kaur, M., Singh, H., Dhillon, J. S., Batra, M., \& Saini, M. (2017). MTA versus Biodentine: Review of Literature with a Comparative Analysis. Journal of clinical and diagnostic research: JCDR, 11(8), ZG01-ZG05.

Keskin, C., Demiryurek, E. O., \& Ozyurek, T. (2015). Color stabilities of calcium silicate-based materials in contact with different irrigation solutions. Journal of endodontics, 41(3), 409-411.

Keskin, C., Sariyilmaz, E., \& KeleS, A. (2019). The effect of bleaching agents on the compressive strength of calcium silicate-based materials. Australian endodontic journal : the journal of the Australian Society of Endodontology Inc, 45(3), 311-316.

Khedmat, S., Dehghan, S., Hadjati, J., Masoumi, F., Nekoofar, M. H., \& Dummer, P. M. (2014). In vitro cytotoxicity of four calcium silicate-based endodontic cements on human monocytes, a colorimetric MTT assay. Restorative dentistry \& endodontics, 39(3), 149-154.

Kim, S., \& Kratchman, S. (2006). Modern endodontic surgery concepts and practice: a review. Journal of endodontics, 32(7), 601-623.

Kontakiotis, E. G., Filippatos, C. G., Tzanetakis, G. N., \& Agrafioti, A. (2015). Regenerative endodontic therapy: a data analysis of clinical protocols. Journal of endodontics, 41(2), 146-154.

Laurent, P., Camps, J., \& About, I. (2012). Biodentine(TM) induces TGF- $\beta 1$ release from human pulp cells and early dental pulp mineralization. International endodontic journal, 45(5), 439-448

Laurent, P., Camps, J., De Méo, M., Déjou, J., \& About, I. (2008). Induction of specific cell responses to a Ca(3)SiO(5)-based posterior restorative material. Dental materials : official publication of the Academy of Dental Materials, 24(11), 1486-1494.

Lee, B. N., Lee, K. N., Koh, J. T., Min, K. S., Chang, H. S., Hwang, I. N., Hwang, Y. C., \& Oh, W. M. (2014). Effects of 3 endodontic bioactive cements on osteogenic differentiation in mesenchymal stem cells. Journal of endodontics, 40(8), 1217-1222.

Ma, J., Shen, Y., Stojicic, S., \& Haapasalo, M. (2011). Biocompatibility of two novel root repair materials. Journal of endodontics, 37(6), $793-798$.

Mori, G. G., Teixeira, L. M., de Oliveira, D. L., Jacomini, L. M., \& da Silva, S. R. (2014). Biocompatibility evaluation of biodentine in subcutaneous tissue of rats. Journal of endodontics, 40(9), 1485-1488.

Możyńska, J., Metlerski, M., Lipski, M., \& Nowicka, A. (2017). Tooth Discoloration Induced by Different Calcium Silicate-based Cements: A Systematic Review of In Vitro Studies. Journal of endodontics, 43(10), 1593-1601.

Murray, P. E., Garcia-Godoy, F., \& Hargreaves, K. M. (2007). Regenerative endodontics: a review of current status and a call for action. Journal of endodontics, 33(4), 377-390.

Naghavi, N., Ghoddusi, J., Sadeghnia, H. R., Asadpour, E., \& Asgary, S. (2014). Genotoxicity and cytotoxicity of mineral trioxide aggregate and calcium enriched mixture cements on L929 mouse fibroblast cells. Dental materials journal, 33(1), 64-69.

Nai, G. A., Logar, G., Mori, G. G., Teixeira, L. M., Silva, B. C., Moraes, A. E., \& Cabral, F. A. (2016). Evaluation of the genotoxicity and mutagenicity of Ca3SiO5-based cement. Brazilian oral research, 30(1), S1806-83242016000100277.

Namour, M., \& Theys, S. (2014). Pulp revascularization of immature permanent teeth: a review of the literature and a proposal of a new clinical protocol. TheScientificWorldJournal, 2014, 737503.

Narang, I., Mittal, N., \& Mishra, N. (2015). A comparative evaluation of the blood clot, platelet-rich plasma, and platelet-rich fibrin in regeneration of necrotic immature permanent teeth: A clinical study. Contemporary clinical dentistry, 6(1), 63-68.

Odabaş, M. E., Bani, M., \& Tirali, R. E. (2013). Shear bond strengths of different adhesive systems to biodentine. TheScientificWorldJournal, $2013,626103$.

Palit Madhu Chanda, Hegde, K. S., Bhat, S. S., Sargod, S. S., Mantha, S., \& Chattopadhyay, S. (2014). Tissue engineering in endodontics: root canal revascularization. The Journal of clinical pediatric dentistry, 38(4), 291-297. 
Patil, U., Yeli, M., Tapashetti, S., Naik, B., \& Tilakchand, M. (2019). Effect of varying durations of intracanal medicament application used in regenerative endodontic treatment on the push-out bond strength of a novel cement: NeoMTA Plus. Journal of conservative dentistry : JCD, $22(1)$, 48-53.

Parirokh, M., Sadr, S., Nakhaee, N., Abbott, P. V., \& Manochehrifar, H. (2014). Comparison between prescription of regular or on-demand ibuprofen on postoperative pain after single-visit root canal treatment of teeth with irreversible pulpitis. Journal of endodontics, 40(2), 151-154.

Parirokh, M., Torabinejad, M., \& Dummer, P. (2018). Mineral trioxide aggregate and other bioactive endodontic cements: an updated overview - part I: vital pulp therapy. International endodontic journal, 51(2), 177-205.

Parirokh, M., \& Torabinejad, M. (2010). Mineral trioxide aggregate: a comprehensive literature review--Part I: chemical, physical, and antibacterial properties. Journal of endodontics, 36(1), 16-27.

Patidar, S., Kalra, N., Khatri, A., \& Tyagi, R. (2017). Clinical and radiographic comparison of platelet-rich fibrin and mineral trioxide aggregate as pulpotomy agents in primary molars. Journal of the Indian Society of Pedodontics and Preventive Dentistry, 35(4), 367-373.

Peters, O. A., Galicia, J., Arias, A., Tolar, M., Ng, E., \& Shin, S. J. (2016). Effects of two calcium silicate cements on cell viability, angiogenic growth factor release and related gene expression in stem cells from the apical papilla. International endodontic journal, 49(12), 1132-1140.

Pradelle-Plasse, N. \& Tran Xuan-Vin, C. (2009). Physico-chemical properties of Biodentine. In: Goldberg, M. editor. Biocompatibility or cytotoxic effects of dental composites.1st ed. Oxford: Coxmoor Publishing, p. 222.

Prati, C., \& Gandolfi, M. G. (2015). Calcium silicate bioactive cements: Biological perspectives and clinical applications. Dental materials: official publication of the Academy of Dental Materials, 31(4), 351-370.

Quintana, R. M., Jardine, A. P., Grechi, T. R., Grazziotin-Soares, R., Ardenghi, D. M., Scarparo, R. K., Grecca, F. S., \& Kopper, P. (2019). Bone tissue reaction, setting time, solubility, and $\mathrm{pH}$ of root repair materials. Clinical oral investigations, 23(3), 1359-1366.

Rajasekharan, S., Martens, L. C., Cauwels, R., \& Anthonappa, R. P. (2018). Biodentine ${ }^{\mathrm{TM}}$ material characteristics and clinical applications: a 3 year literature review and update. European archives of paediatric dentistry: official journal of the European Academy of Paediatric Dentistry, 19(1), 1-22.

Roberts, H. W., Toth, J. M., Berzins, D. W., \& Charlton, D. G. (2008). Mineral trioxide aggregate material use in endodontic treatment: a review of the literature. Dental materials : official publication of the Academy of Dental Materials, 24(2), 149-164.

Saeki, K., Fujita, Y., Shiono, Y., Morimoto, Y., \& Maki, K. (2014). Pulp revascularization in immature permanent tooth with apical periodontitis using mineral trioxide aggregate. Case reports in medicine, 2014, 564908.

Sawyer, A. N., Nikonov, S. Y., Pancio, A. K., Niu, L. N., Agee, K. A., Loushine, R. J., Weller, R. N., Pashley, D. H., \& Tay, F. R. (2012). Effects of calcium silicate-based materials on the flexural properties of dentin. Journal of endodontics, 38(5), 680-683.

Siboni, F., Taddei, P., Prati, C., \& Gandolfi, M. G. (2017). Properties of NeoMTA Plus and MTA Plus cements for endodontics. International endodontic journal, 50(2), e83-e94

Silva, E. J., Senna, P. M., De-Deus, G., \& Zaia, A. A. (2016). Cytocompatibility of Biodentine using a three-dimensional cell culture model. International endodontic journal, 49(6), 574-580.

Simsek, N., Alan, H., Ahmetoglu, F., Taslidere, E., Bulut, E. T., \& Keles, A. (2015). Assessment of the biocompatibility of mineral trioxide aggregate, bioaggregate, and biodentine in the subcutaneous tissue of rats. Nigerian journal of clinical practice, 18(6), 739-743.

Songtrakul, K., Azarpajouh, T., Malek, M., Sigurdsson, A., Kahler, B., \& Lin, L. M. (2020). Modified Apexification Procedure for Immature Permanent Teeth with a Necrotic Pulp/Apical Periodontitis: A Case Series. Journal of endodontics, 46(1), 116-123.

Tanomaru-Filho, M., Andrade, A. S., Rodrigues, E. M., Viola, K. S., Faria, G., Camilleri, J., Guerreiro-Tanomaru, J. M. (2017). Biocompatibility and mineralized nodule formation of Neo MTA Plus and an experimental tricalcium silicate cement containing tantalum oxide. Int Endod J. 50, e31-e39.

Tomás-Catalá, C. J., Collado-González, M., García-Bernal, D., Oñate-Sánchez, R. E., Forner, L., Llena, C., Lozano, A., Moraleda, J. M., \& RodríguezLozano, F. J. (2018). Biocompatibility of New Pulp-capping Materials NeoMTA Plus, MTA Repair HP, and Biodentine on Human Dental Pulp Stem Cells. Journal of endodontics, 44(1), 126-132.

Torabinejad, M., \& Parirokh, M. (2010). Mineral trioxide aggregate: a comprehensive literature review--part II: leakage and biocompatibility investigations. Journal of endodontics, 36(2), 190-202.

Torabinejad, M., Parirokh, M., \& Dummer, P. (2018). Mineral trioxide aggregate and other bioactive endodontic cements: an updated overview - part II: other clinical applications and complications. International endodontic journal, 51(3), 284-317.

Ürkmez, E. Ş., \& Pınar Erdem, A. (2020). Bioactivity evaluation of calcium silicate-based endodontic materials used for apexification. Australian endodontic journal: the journal of the Australian Society of Endodontology Inc, 46(1), 60-67.

Zanini, M., Sautier, J. M., Berdal, A., \& Simon, S. (2012). Biodentine induces immortalized murine pulp cell differentiation into odontoblast-like cells and stimulates biomineralization. Journal of endodontics, 38(9), 1220-1226.

Zhou, W., Zheng, Q., Tan, X., Song, D., Zhang, L., \& Huang, D. (2017). Comparison of Mineral Trioxide Aggregate and iRoot BP Plus Root Repair Material as Root-end Filling Materials in Endodontic Microsurgery: A Prospective Randomized Controlled Study. Journal of endodontics, 43(1), 1-6.

Zhu, X., Zhang, C., Huang, G. T., Cheung, G. S., Dissanayaka, W. L., \& Zhu, W. (2012). Transplantation of dental pulp stem cells and platelet-rich plasma for pulp regeneration. Journal of endodontics, 38(12), 1604-1609. 